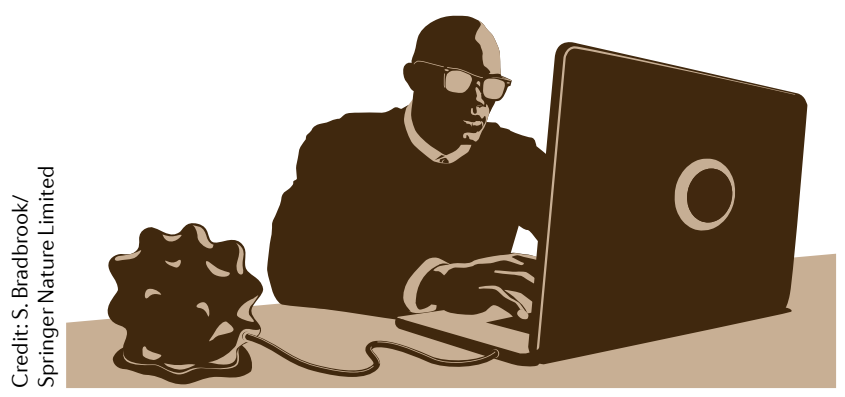

was dependent on STAT6 and PI3K-mTORC2-AKT signalling activity.

Finally, tumour progression was shown to be impaired in mice with a myeloid cell-restricted deficiency in the cholesterol and lipid transporters ABCA1 and ABCG1, and TAMs from cholesterol efflux these mice showed reduced expression of IL-4-associated genes and an pathways could be targeted therapeutically to enhance the pro-tumour activity of macrophages upregulation of tumoricidal genes.

The authors propose that cholesterol efflux pathways could be targeted therapeutically to enhance the pro-tumour activity of macrophages.

Yvonne Bordon

ORIGINAL ARTICLE Goossens, P. et al. Membrane cholesterol efflux drives tumorassociated macrophage reprogramming and tumor progression. Cell Metab. https://doi.org/ 10.1016/j.cmet.2019.02.016 (2019)

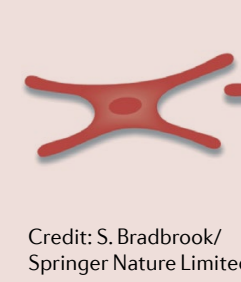

of the cell cycle, showed metabolic stress and were more prone to undergo apoptosis. This was linked to defects in energy metabolism, and IL-17 was found to promote glucose uptake, glycolysis and oxidative phosphorylation in FRCs in

inflamed lymph nodes.

IL-17 alone did not increase metabolic activity in FRCs but instead acted on FRCs primed by other inflammatory signals. The authors showed that IL-17 induction of $I \kappa B \zeta$ supports FRC metabolism by promoting glucose uptake and expression of CPT1A, a rate-limiting

IL- 17 was found to promote glucose uptake, glycolysis and oxidative phosphorylation in FRCs in enzyme of mitochondrial fatty acid oxidation. They speculate that the ability of IL-17 to heighten metabolic activity in FRCs may partly explain its link to the loss of tolerance and disease relapse in autoimmunity. Yvonne Bordon inflamed lymph nodes

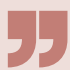

ORIGINAL ARTICLE Majumder, S. et al. IL-17 metabolically reprograms activated fibroblastic reticular cells for proliferation and survival. Nat. Immunol. https://doi.org/10.1038/s41590019-0367-4 (2019)

\section{Non-inflammatory effects of liver macrophages}

Previous studies have suggested that in metabolic diseases - such as obesityassociated insulin resistance liver macrophages acquire a pro-inflammatory phenotype. However, the modest metabolic effects of anti-inflammatory drugs have brought this into question. A new study in Nature Metabolism shows, instead, that liver macrophages

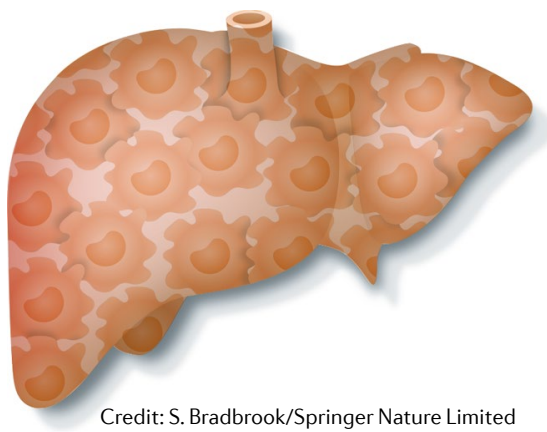
produce the non-inflammatory factor insulin-like growth factor-binding protein 7 (IGFBP7) to directly regulate liver metabolism.

RNA sequencing of liver macrophages from lean individuals and insulin-sensitive or insulin-resistant obese individuals showed no differences in the expression of inflammatory markers. In mice, the expression of tumour necrosis factor (Tnf) in liver macrophages was increased only after 12 weeks of a high-fat diet (HFD), concurrent with the recruitment of liver-infiltrating macrophages. Thus, activation of resident liver macrophages to a pro-inflammatory phenotype does not seem to be required for the initiation of metabolic disease; rather, liver inflammation in the longer term results from the recruitment of macrophages from the circulation.

Using glucan-encapsulated RNA interference (RNAi) particles to silence the expression of Rela - a major regulator of inflammatory responses through $N F-\kappa B$ signalling - specifically in liver macrophages of obese mice, the authors showed that the transcription of inflammatory cytokine genes was unaffected despite improved glucose tolerance. Thus, the role of liver macrophages in decreasing insulin sensitivity during obesity does not seem to involve a pro-inflammatory phenotypic switch and the results suggest the existence of an alternative NF- $\kappa \mathrm{B}$-dependent pathway.

Further transcriptome analysis showed that lgfbp7 is the most highly upregulated gene in liver macrophages from HFD-fed mice compared with mice fed a normal diet. In humans, IGFBP7 expression in liver macrophages was unchanged by obesity or insulin resistance. Instead, adenosine-to-inosine RNA editing of IGFBP7 - which leads to an amino acid substitution in the protein - was present in $90 \%$ of insulin-resistant obese individuals compared with $30 \%$ of insulin-sensitive obese individuals or lean controls. The edited form of IGFBP7 bound to the insulin receptor with greater affinity than wild-type IGFBP7.

lgfbp7 silencing in liver macrophages of obese mice significantly reduced overnight fasting hyperglycaemia and reduced the accumulation of triglycerides in the liver (hepatic steatosis). The authors showed that binding of IGFBP7 to the insulin receptor activates different insulin-induced signalling pathways in insulin-sensitive compared with insulin-resistant hepatocytes; this differential signalling mediates the switch from decreased to increased expression of gluconeogenic and lipogenic genes.

The results suggest that targeting liver macrophages directly rather than targeting inflammation could be a more effective strategy in metabolic disease.

Kirsty Minton 